OPEN ACCESS

Edited by:

Argyrios Tzouvelekis,

Alexander Fleming Biomedical Sciences Research Center, Greece

Reviewed by:

Koji Sakamoto,

Nagoya University, Japan

Gunnar N. Hillerdal,

Karolinska University

Hospital, Sweden

*Correspondence:

Bogdan Grigoriu

bogdan.grigoriu@bordet.be

Specialty section:

This article was submitted to

Pulmonary Medicine,

a section of the journal

Frontiers in Medicine

Received: 17 January 2020

Accepted: 30 March 2020

Published: 07 May 2020

Citation:

Coureau M, Meert A-P, Berghmans T and Grigoriu B (2020) Efficacy and

Toxicity of Immune -Checkpoint Inhibitors in Patients With Preexisting

Autoimmune Disorders.

Front. Med. 7:137.

doi: 10.3389/fmed.2020.00137

\section{Efficacy and Toxicity of Immune -Checkpoint Inhibitors in Patients With Preexisting Autoimmune Disorders}

\author{
Michelle Coureau ${ }^{1}$, Anne-Pascale Meert ${ }^{1}$, Thierry Berghmans ${ }^{2}$ and Bogdan Grigoriu ${ }^{\text {* }}$ \\ ${ }^{1}$ Service des Soins Intensifs et Urgences Oncologiques, Institut Jules Bordet, Université Libre de Bruxelles (ULB), Brussels, \\ Belgium, 2 Service d'Oncologie Medicale, Unité d'Oncologie Thoracique, Institut Jules Bordet, Université Libre de Bruxelles \\ (ULB), Brussels, Belgium
}

Immunotherapy is an important armamentarium for cancer treatment nowadays. Apart from their significant effectiveness in controlling disease they also generate potential severe immune related adverse effects. Preexistence of immune related conditions may eventually predispose to the development of more severe complication and extreme caution have been taken in treating these patients. We performed a literature review searching for case reports and case series in order to offer evidence-based data for clinical management of these patients. Preexisting serological-only immune abnormalities or presence of a predisposing genetic background does not seem to confer significant risk but existing data is scarce. Most patients with preexistent autoimmune diseases can probably treated with checkpoint inhibitors as they seem to have at least the same response rate as the general cancer population. Under treatment, a significant part of them (at least 30\%) can experience a flare of their baseline disease which can sometime be severe. Life-threatening cases seems rare and disease flare can be generally managed with steroids. The volume of available data is more important for rheumatologic diseases than for inflammatory bowel diseases were more caution should be observed. However, it has to be kept in mind that new immune related adverse effects (IrAE) are seen with a similar frequency as the flare of the baseline disease. Both flare-up's and newly developed IrAE are generally manageable with a careful clinical follow-up and prompt therapy.

Keywords: cancer, immunotherapy, autoimmunity, side-effect, flare-up

\section{INTRODUCTION}

Immunotherapy has become a cornerstone in cancer treatment offering unexpected treatment opportunities for patients with metastatic, loco-regional, or even operable tumors in melanoma, lung, urothelial, and a number of other tumoral localizations.

Two main directions of intervention are now clinically available for manipulating immune checkpoints by either targeting CTLA-4 or PD-1/PD-L1. Our current understanding of the role of these two molecules is that anti CTLA- 4 is involved in the lymphocyte proliferation process after antigen specific T-cell receptor activation while anti PD-1/PD-L1 act predominantly in peripheral tissues during the effector step. However, CTLA-4 is also expressed on regulatory T lymphocytes and is thus involved in peripheral inhibition of $\mathrm{T}$ cell proliferation. 
Despite the effectiveness of these products, two main issues arise from theoretical and practical considerations.

First not all patients respond to therapy. To date prediction of response to immune checkpoint inhibitors is difficult and available predictive markers are not optimal. Secondly cancer may be associated with a number of autoimmune manifestations either as a paraneoplastic condition (1) or as comorbidities. In experimental models, knocking-out CTLA-4 results in a massive proliferation and lymphocytic infiltration of tissues (2) while PD-1 knock-down mice are prone to autoimmune disease [reviewed in (3)]. These phenotypes can also be found in humans with CTLA-4 (4, 5) or PD-L1 (6) deficiency. Nevertheless, the mechanisms involved in antitumor immune response induced by checkpoint inhibitors may be different from those of autoimmune disease. However, it is clear that using checkpoint inhibitors to treat cancer poses the risk of a flare up of preexistent autoimmune disease and the development of new autoimmune manifestations in patients with preexisting autoimmunity even if present as a biological only manifestation (as for example positive auto antibodies).

Thus, these patients with preexisting autoimmune manifestations have been traditionally excluded from clinical trials as it was expected to have severe autoimmune manifestations which could exceed the potential benefit from tumor control. This subgroup of cancer patients harboring autoimmune manifestations is not trivial. Depending on the definition used, a large register-based analysis (SEER) including 2,10,509 patients with lung cancer identified between $13.5 \%$ (more restrictive definition) and 24.6\% (more liberal definition) of cancer patients as having an autoimmune disease of any type (7). The most frequent manifestations found were rheumatoid arthritis (5.9\%), psoriasis (2.8\%), polymyalgia rheumatica (1.8\%), Addison disease (1\%), systemic lupus erythematosus (SLE- $-0.9 \%)$, ulcerative colitis $(0.8 \%)$, giant cell arteritis $(0.8 \%)$, sicca syndrome $(0.6 \%)$, regional enteritis $(0.5 \%)$, and Meniere disease $(0.5 \%)$. However, these autoimmune manifestations can be very broad as it has been recently reviewed for lung cancer in a series of articles curated by Jean Paul Scuiler (1, 8-11).

Thus, it is useful to perform, prior to any treatment, a careful evaluation of the possible autoimmune comorbidities in order to avoid possible harm, evaluate risk/benefit ratios, and anticipate possible complications.

\section{PATIENTS AT RISK BUT WITHOUT PREVIOUS CLINICAL EVIDENCE OF AUTOIMMUNE DISEASE}

In patient with lung cancer receiving checkpoint inhibitors, development of diverse IrAE is not completely random. Despite the fact that the incidence of IrAE increase with treatment duration, there is a pattern of developing specific organ manifestations in defined time frames. Thus, one can postulate that these IrAE develop in patients caring specific genotypes or having a previous immune priming predisposing for developing IrAE. Very little is known on this matter. Hasan Ali et al. (12) reported on a small series of 102 patients receiving anti PD1/PD-L1 and anti CTLA-4 therapies, a very strong correlation between the presence of pruritus and the HLADRB1*11:01 allele. Patient carrying these allele not only develop more pruritus than controls but also developed it earlier. Interesting, in previous investigations this specific allele has been associated with the development of atopic dermatitis in a small cohort of 185 children of Korean origin (13). The same allele has been associated with systemic sclerosis with anti-topoisomerase antibodies (14) or with the risk of developing systemic juvenile arthritis (15) or atopic dermatitis (13). The same Swiss group of Hasan Ali et al. also demonstrated an association trend between HLA DBQ1*03:01 allele and the development of colitis. Again, this allele has been previously associated firmly with the development of inflammatory bowel disease (16). Similarly it is possible that some Single Nucleotide Polymorphism (SNP's), which are associated with a higher risk of autoimmune disease (colitis, diabetes, and allergy), positively impact the response rate to anti PD-1 therapy (17).

A systematic review of susceptible loci associated with development of IrAE's under checkpoint inhibitor therapy has been recently performed by Hoefsmit et al. (18) with a compartmentalized analysis based on affected organ and type of adverse effect. A huge number of susceptible loci, with a very variable physiologic/pathologic role, have been identified. However, these data can be hypothesis generating at most. At present time there is no sound information that can be applied to practice and there is no scientific basis to withhold checkpoint inhibitor treatment in people considered at risk based on these criteria.

Yoneshima et al. analyzed if pretreatment positive antinuclear antibodies (ANA) are associated either with IrAE or treatment response after administration of PD-1 inhibitors (19). They analyzed a group of 83 patients, of which 18 had preexisting positive serum ANA at a titer of 1:40 or higher. There was no statistical relationship between the presence of ANA and the risk of developing IrAE but there was an association trend between increasing ANA titers and the development of IrAE. Overall, response and disease control rates were similar in patients with or without ANA but there was also a higher PFS and OS in patients with negative ANA. A similar investigation was conducted by Sakakida et al. (20) which followed 191 patients treated with nivolumab, pembrolizumab, atezolizumab, or durvalumab and used a cutoff titer for positive ANA at 1:160. There was no significant difference in the development of IrAE between ANA positive and ANA negative patients with the exception of patients who developed colitis which had higher ANA titers than those who do not. Again, an elevated ANA titer was associated with a numerically lower disease control rate (37.5 and 67.5\%) but this was not statistically significant $(p=0.08)$. Furthermore, for both series, the global numbers are rather small and the significance (both from statistical and clinical point of view) is debatable. Moreover, there are case reports of serious IrAE (i.e., fatal pneumonitis) that followed immune checkpoint administration in patients with pre-existing anti-synthetase syndrome (21).

To date, there is no sufficient data to warrant a systematic search for autoantibodies in cancer patients that are planned 
to receive checkpoint inhibitors. However, if knowledge of such positive finding is available, a clinical surveillance is warranted in order to identify, as early as possible, autoimmune manifestations associated with these autoantibodies.

\section{PEOPLE WITH PREEXISTING CLINICALLY DIAGNOSED AUTOIMMUNE DISEASE}

Since these patients have been systematically excluded from clinical trials, subgroup analysis is scarcely available in the literature. Existing data originate from case reports, retrospective analyses of small series or registry data. Only one subgroup analysis of randomized trials is available but it does not cover the entire spectrum of autoimmune disease encountered in practice.

\section{Case Reports}

A number of 37 publications reporting 41 cases have been found [(22-59); Table 1]. Most cases were metastatic melanomas $(n=$ 32), five non-small cell lung cancers, two Merkel cell carcinoma, one urothelial carcinoma, one colon cancer. These 41 cases received 44 immunotherapy treatments namely ipilimumab ( $n$ $=15)$, one reinduction ipilimumab, nivolumab $(n=9)$, or pembrolizumab $(n=18)$, one unclear, which globally reflect the successive market authorizations of these drugs. No reports combining immunotherapy with chemotherapy were found. Pre-existing autoimmune manifestations consisted of Crohn disease/ulcerative colitis $(n=8)$, rheumatoid arthritis $(n=$ $7)$, psoriasis $(n=8)$, myasthenia gravis $(n=-6)$, multiple sclerosis $(n=3)$, Hashimoto thyroiditis $(n=2)$, and one each of lupus, sarcoidosis, immune thrombocytopenia, melanoma associated retinopathy, Churg Strauss syndrome, granulomatosis with polyangiitis, hypothyroidy and type 1 diabetes, Bechet disease, and bullous pemphigoid. Globally a tumor effect of immunotherapy is reported for 40 out of the 44 treatments with six complete responses, 27 partial responses, three stable disease and four progressions. The response rate is $82.5 \%$ and the disease control rate is $90 \%$ which are very high figures but is it highly probable that this reflects a strong publication bias. In a majority of cases $(n=29$, i.e., $65.6 \%)$ immunotherapy resulted in a flare up of the baseline disease which was managed with steroids or infliximab, adalimumab, or rituximab. In 10 cases (i.e., 22.7\%) flare ups were considered severe/very severe with one death. In another case, a flare up of colitis was infliximab resistant and the patient subsequently developed a toxic epidermal necrosis and died. Only 16 cases had no flare up of the base line disease. Six of these patients developed other IrAE as pneumonitis, toxic epidermal necrosis, acute colitis, psoriasis, vasculitic neuropathy or acute interstitial nephritis. Overall, mortality due to disease flareup is about $4.5 \%$ and very severe (grade 4) toxicities unresponsive to steroids or even additional anti TNF alpha therapy was not uncommon. In only 12 cases no flare-up and no subsequent IrAE developed.

\section{Data From Clinical Trials}

Even if patients with previous autoimmune disease have been excluded from clinical trials a number of patients have been nevertheless included. The FDA made a post-hoc analysis based on aggregate data from 22 trials with four Anti PD$1 /$ PDL-1 agents that was published only in an abstract form (60). The series mainly include thyroid disease and cutaneous autoimmune manifestations (psoriasis and vitiligo)- $-54.5 \%$ of cases. Worsening of the underlying disease occurred in 6-16 percent of the cases. Two grade 4 hyperglycemia were found in patients with preexisting diabetes, as well as three grade 3 hypothyroidy, psoriasis, and interstitial lung disease and one grade 3 ankylosing spondylitis. Only $8-9 \%$ of cases needed steroid prescription and duration of treatment varied between 145 and 196 days. Thus, in this population with probably a strong bias toward non-severe autoimmune manifestations, there are no consequent safety signals identified and administration of PD-1/PDL-1 inhibitors seems safe.

However, the report lacks data concerning the exact type of autoimmune condition for a large proportion of patients. Data on severity and treatment of baseline disease is also scarce and thus these results are to be interpreted with caution.

\section{Case Series}

We found five retrospective series in patients with melanoma (61-65) totalizing 150 cases with autoimmune disease previous to any treatment and 117 with autoimmune manifestations due to a previous therapy with ipilimumab which received another checkpoint inhibitor afterwards. In the series receiving only ipilimumab $(61,63,64)$ the response rates varied between 12 and $50 \%$ (higher for smaller series) while in series with anti PD-1/PDL-1 $(62,65)$ the response rate was around $30 \%$ which is in line with what was described with these drugs in clinical trials. Between 27 and $50 \%$ of cases had a flare up of their baseline autoimmune condition while up to $30 \%$ of patients experienced the development of a new autoimmune toxicity. Grossly, the frequency of flare ups is similar to that of newly developed immune related manifestations. Severity of flare-ups was generally moderate, with $50 \%$ being more than grade one and no very severe cases (grade 4/5). Interestingly, in cases with an ipilimumab induced autoimmune disease, a flare up is rarely seen when subsequently treated with a PD-1/PDL-1 inhibitor. It seems that the presence of an autoimmune manifestation under CTLA-4 inhibition does not preclude anti PD-1/PDL-1 treatment but data in this area is still scare in order to make a firm clinical recommendation. Two case series of the same author $(66,67)$ reported 100 NSCLC cases and another one (68) 112 cases in multiple tumor types.

In these three series the tumor response rates varied between 45 and $51 \%$ while disease flare is seen in $17-47 \%$ of patients. Most of flare-ups are moderate (grade 3 or less). Between 25 and $42 \%$ of patients experienced a new IrAE, not related to the baseline autoimmune disease, during the course of treatment.

The reports do not always include detailed data concerning treatment received for the baseline autoimmune disease and thus it is difficult to derive clinical rules on which patients would eventually develop or not a flare-up of the baseline disease.

A number of 5 cases series with matched controls receiving the same immune checkpoint inhibitor but without autoimmune disease were published (69-73); these series included a total of 701 patients with a very large panel of autoimmune disorders 
TABLE 1 | List of case report of Checkpoint inhibitors in patients with preexisting autoimmune diseases.

\begin{tabular}{|c|c|c|c|c|c|c|}
\hline References & $\begin{array}{l}\text { Pre-existing autoimmune } \\
\text { disease }\end{array}$ & Immunosuppressive treatment & $\begin{array}{l}\text { Cancer } \\
\text { type }\end{array}$ & Drug received & Response & $\begin{array}{l}\text { Disease flare Yes/No new autoimmune } \\
\text { manifestation (treatment) }\end{array}$ \\
\hline Gerdes et al. (31) & Multiple sclerosis & No (minimal radiologic only disease) & Melanoma & Ipi & Yes (PR) & Yes (massive flare) \\
\hline Gettings et al. (32) & Multiple sclerosis & - & Melanoma & Ipi & Yes (PR) & Yes (Severe Flare) \\
\hline \multirow{2}{*}{$\begin{array}{l}\text { Kyi et al. (38) } \\
N=2\end{array}$} & Multiple sclerosis & IFN beta & Melanoma & Ipi & No & No \\
\hline & Rheumatoid arthritis & MTX +PDN & Melanoma & Ipi & Yes (PR) & No \\
\hline \multirow[t]{2}{*}{ Benson et al. (25) } & Rheumatoid arthritis & Etanercept & Melanoma & Ipi & No & $\begin{array}{l}\text { Yes } \\
\text { Acute interstitialnephritis }\end{array}$ \\
\hline & & & & Pem & Yes (PR) & $\begin{array}{l}\text { Yes } \\
\text { (hydroxychloroquine + prednisone) }\end{array}$ \\
\hline Puri and Homsi (48) & Rheumatoid Arthritis & PDN low dose & Melanoma & Pem & Yes (CR) & No \\
\hline \multirow[t]{2}{*}{ Aya et al. (23) } & Rheumatoid arthritis & No & Melanoma & Ipi & Yes (CR) & No Colitis(infliximab) \\
\hline & & & & Pem & Yes (PR) & No Vasculiticneuropathy \\
\hline Hedge et al. (34) & Rheumatoid arthritis & No & Melanoma & Pem & Yes (PR) & - \\
\hline Kageyama et al. (35) & Rheumatoid arthritis & Salazopyrine & Melanoma & Nivo & Yes (CR) & No \\
\hline Zhu and Li (57) & $\begin{array}{l}\text { Rheumatoid arthritis and } \\
\text { Myasthenia gravis }\end{array}$ & No & Melanoma & Pem & Yes (PR) & Yes (Ivlg, plasmapheresis, PDN) \\
\hline Zaremba et al. (56) & Myasthenia gravis & $\begin{array}{l}\text { AZT (followed by MMF and } \\
\text { cyclosporine) }\end{array}$ & $\begin{array}{l}\text { Merkel cell } \\
\text { carcinoma }\end{array}$ & Pem & Yes (PR) & No \\
\hline Lau et al. (39) & Myasthenia gravis & AZT & Melanoma & Pem & - & Yes (steroids + Ivlg) \\
\hline Maeda et al. (40) & Myasthenia gravis & PDN & Melanoma & Nivo & Yes (PR) & Yes (Self-limited) \\
\hline Cooper et al. (28) & Myasthenia gravis & No (pyridostigmine) & NSCLC & Nivo & Yes (PR) & Yes (severe - death) \\
\hline \multirow{2}{*}{$\begin{array}{l}\text { Phadke et al. (46) } \\
N=2\end{array}$} & Myasthenia gravis & MMF & Melanoma & Pem & Yes (PR) & Yes (Grade 4) \\
\hline & Psoriasis/psoriatic arthritis & MTX & Melanoma & Pem & Yes (CR) & Yes \\
\hline Roche et al. (49) & Psoriasis/psoriatic arthritis & - & $\begin{array}{l}\text { Merkel cell } \\
\text { carcinoma }\end{array}$ & Pem & Yes (PR) & No \\
\hline Sahuquillo-Torralba et al. (50) & Psoriasis & $\begin{array}{l}\text { Local treatment Psoriasis severity } \\
\text { score } 3 \text { on } 5 \% \text { onBSA }\end{array}$ & NSCLC & Pem & Yes (PR) & $\begin{array}{l}\text { Yes (severe - Psoriasis score } 22 \text { on } 81 \% \text { of BSA; } \\
\text { Pem continued and resolution with severity score } 4 \\
\text { after acitretin } 35 \mathrm{mg} / \mathrm{d} \text { ) }\end{array}$ \\
\hline De Bock et al. (58) & Psoriasis & No & Melanoma & $\begin{array}{l}\text { Nivo (previous } \\
\text { Ipi) }\end{array}$ & Yes (SD) & Yes (local steroids and every 3 week Nivo) \\
\hline Chia and John (27) & Psoriasis & No & NSCLC & - & - & Yes (severe flare) \\
\hline Kato et al. (37) & Psoriasis & No & Melanoma & Nivo & - & Yes \\
\hline Matsumura et al. (41) & Psoriasis & No & Melanoma & Nivo & Yes (PR) & $\begin{array}{l}\text { Yes } \\
\text { Pneumonitis }\end{array}$ \\
\hline Esfahani and Miller (29) & Psoriasis+ Crohn disease & No & Colon & Pem & Yes (PR) & Yes Psoriasis and gastrointestinal \\
\hline Frohne et al. (30) & Crohn disease & $\begin{array}{l}\text { Infliximab/AZT } \\
\text { followed by Vedolizumab }\end{array}$ & Melanoma & Pem & Yes (CR) & No \\
\hline
\end{tabular}


TABLE 1 | Continued

\begin{tabular}{|c|c|c|c|c|c|c|}
\hline References & $\begin{array}{l}\text { Pre-existing autoimmune } \\
\text { disease }\end{array}$ & Immunosuppressive treatment & $\begin{array}{l}\text { Cancer } \\
\text { type }\end{array}$ & Drug received & Response & $\begin{array}{l}\text { Disease flare Yes/No new autoimmune } \\
\text { manifestation (treatment) }\end{array}$ \\
\hline Uemura et al. (53) & Crohn disease & Tocilizumab & Melanoma & Pem & Yes (PR) & Yes (adalimumab) \\
\hline Gielisse and de Boer (33) & Crohn disease & No & Melanoma & Ipi & Yes (PR) & Yes (manageable) \\
\hline Kamil et al. (36) & Crohn disease & Mesalazine and PDN & NSCLC & Ipi & Yes (PR) & $\begin{array}{l}\text { Yes (infliximab resistant) } \\
\text { Toxic epidermal necrosis (death) }\end{array}$ \\
\hline \multirow[t]{2}{*}{ Bostwick et al. (26) } & Ulcerative colitis & No & Melanoma & Ipi & Yes (PR) & Yes (infliximab) \\
\hline & & $\mathrm{AZT}+\mathrm{PDN}$ & & Reinduction Ipi & Yes (CR) & $\begin{array}{l}\text { Yes } \\
\text { Also new auto immunedisease }\end{array}$ \\
\hline \multirow{2}{*}{$\begin{array}{l}\text { Pedersen et al. (45) } \\
N=2\end{array}$} & Ulcerative colitis & Infliximab & Melanoma & Ipi & Yes (PR) & No \\
\hline & Bechet disease & - & Melanoma & Ipi & No & No \\
\hline $\begin{array}{l}\text { Plachouri et al. (47) } \\
\text { Wieshaupt and Sunderkötter } \\
\text { (54) } \\
\text { (duplicatepublication) }\end{array}$ & Sarcoidosis & PDN low dose & Melanoma & Ipi & No & Yes (Flare with muscular involvement) \\
\hline Audemard et al. (22) & $\begin{array}{l}\text { Melanoma Associated } \\
\text { Retinopathy }\end{array}$ & No & Melanoma & Ipi & Yes (PR) & No (diminishing symptoms secondary to response) \\
\hline \multirow[t]{2}{*}{ Beck et al. (24) } & Bullous pemphigoid & No & Melanoma & Ipi & Yes (SD) & Yes - steroids \\
\hline & & & & Pem & Yes (SD) & Yes - steroids \\
\hline Maul et al. (42) & $\begin{array}{l}\text { Churg Strauss + Ipi induced } \\
\text { colitis }\end{array}$ & - & Melanoma & Pem & Yes (PR) & No \\
\hline Nabel et al. (43) & $\begin{array}{l}\text { GPA (granulomatosis with } \\
\text { polyangiitis) }\end{array}$ & Cyclophosphamide PTX, PDN & $\begin{array}{l}\text { MEN2+ } \\
\text { Urothelial } \\
\text { carcinoma }\end{array}$ & Pem & Yes (PR) & Yes: PDN +rituximab \\
\hline Tagliamento et al. (52) & $\mathrm{SLE}+\mathrm{HCV}$ & $\begin{array}{l}\text { PDN } 5 \text { mg } \\
\text { HCQ 200mg }\end{array}$ & Melanoma & Nivo & Yes (PR) & No \\
\hline Stephen Bagley et al. (51) & Immune thrombocytopenia & No & NSCLC & Nivo & Yes (PR) & Yes (mild) \\
\hline Akturk et al. (59) & $\begin{array}{l}\text { Hypothyroidism } \\
\text { Type } 1 \text { diabetes } \\
\text { (+renal graft) }\end{array}$ & $\begin{array}{l}\text { Immuno suppression (PDN, MMF, } \\
\text { Tacro-graft) }\end{array}$ & Melanoma & Pem + Nivo & - & $\begin{array}{l}\text { Yes (graft rejection) Diabetic ketoacidosis and more } \\
\text { severe hypothyroidia }\end{array}$ \\
\hline $\begin{array}{l}\text { Narita et al. (44) } \\
N=2\end{array}$ & Hashimoto thyroiditis & No & Melanoma & Nivo & Yes (PR) & Yes (serological) \\
\hline Yonezaki et al. (55) & Hashimoto thyroiditis & - & Melanoma & Ipi (after Nivo) & Yes (PR) & Yes thyroid storm \\
\hline
\end{tabular}

HCQ, hidroxycloroquine; PDN, prednisone; AZT, azathioprine; MMF, mycophenolate mofetil; CycloA, ciclosporin A; Nivo, nivolumab; Pem, pembrolizumab; Ipi, ipilimumab; HCV, hepatitis virus C; SLE, systemic lupus erythematosus. 
TABLE 2 | Case series with preexisting autoimmune disease (AD) treated with checkpoint inhibitors.

\begin{tabular}{lllll}
\hline References & Tumor & $N=$ & Baseline autoimmune dis & Benefit
\end{tabular}

\section{Post-hoc analysis of clinical trial data}

Weinstock et al. (60)

$\begin{array}{ll}N=552 \quad & \text { Thyroid } n=188-34 \% \\ & \text { Psoriasis } n=70-12.5 \% \\ & \text { Vitiligo } n=44-8 \%\end{array}$

Other $=250$

Retrospective case series

Melanoma

$N=8$

$\operatorname{RA}(n=8)$

$50 \%$ MTX and/or AntiTNFa

Johnson et al. (63) Melanoma

$$
N=30
$$

Rheumatoid arthritis $n=6$

Crohn/ulcerative colitis $n=6$

Psoriasis $n=5$

Multiple sclerosis $n=2$

Lupus $n=2$

Sarcoidosis $n=2$

Thyroiditis $n=3$

Other $n=7$

Kahler et al. (64)

Melanoma

$N=41$ (with 44

$\mathrm{AD}$ conditions)

Thyroiditis $n$

disease/ulcerative colitis $n=3$, neurological $n$

$=2$, sarcoidosis $n=2$, pancreatitis $n=1$

$n=11$ on immunosuppressors(25\%)

Menzies et al. (62) Melanoma

$N=119$
95 with IrAE
after Ipi
$N=52$
preexisting $A D$

reported

$29 \%$ AD active symptoms

preexisting $A D$

Gutzmer et al. (65) Melanoma $\quad N=19$

Thyroiditis $n=6$

Psoriasis $n=3$

Spondylarthritis/ankylosing spondylitis/

Myositis/polymyalgia/rheumatoid arthritis $n=6$

Sarcoidosis $n=2$

Multiple sclerosis/Guillain Barré/Ulcerative

colitis/Churg Strauss $n=1$ each

$N=22$

IrAE induced by Ipi

Nivo $41 \%$

Colitis $n=11(\operatorname{Gr} 3=7 \mathrm{GR} 4=1)$

Pem 59\%
Anti PD-1/PDL-1

(no details)

Ipi

\section{Pem $91.5 \%$}

Nivo $8.5 \%$

Nivo 63\%
Pem 37\%

$32 \% \mathrm{PR}$

$10 \%$ SD

$58 \%$ PD days)

2 CR (25\%)

$2 \mathrm{PR}$

$3 \mathrm{SD}$

$1 \mathrm{PD}$

on Ipi

No data (mean duration of Worsening of baseline disease between 6 and

treatment between 145 and 196 16\%. Two grade 4 hyperglycemia and 7 grade

3. Steroids required in $8-9 \%$ of patients

$17 \%$ experienced anylrAE

Stable disease $n=3(10 \%)$

$62.5 \%$ treat discontinuation

Median 2 cycles

Dis flare $50 \%$ grade $125 \%$

$50 \%$ more than grade 1

Grade 3 IrAE (All G3colitis)

$27 \%$ had a flare -up

No severe cases

$33 \%$ had an IrAE (Colitis, Thyroiditis,

Hypophysitis)

$1 \mathrm{CR}, 4 \mathrm{PR} \geq 12 \%$ response rate $N=12(29.2 \%)$ resulted in a flare up of preexisting disease

$N=12(29.2 \%)$ had a new IrAE

$N=23(56 \%)$ had no flare/no new IrAE

Response rate 33\% in Flare up in 38\%:

preexisting $A D$ and $40 \%$ if IrAE $\quad$-RA 54\%

-Polymyalgia 100\%

-SJögren, thrombocytopenic purpura, 100\% -No flare if Crohn or neurological disease

$29 \%$ developed new adverse effects

No deaths

$42 \%$ had flare up in 3-20 weeks

-Rhumatological disease: $55 \%$

-Colitis 100\%

-Psoriasis 50\%

-Thyroiditis20\%

$16 \%$ had a new IrAE

Only 1/22 Flare-up (5\%)

$23 \%$ developed another IrAE

Thyroiditis $n=3$

Hepatitis $n=1$ 
TABLE 2 | Continued

\begin{tabular}{|c|c|c|c|c|c|c|}
\hline References & Tumor & $N=$ & Baseline autoimmune dis & Drug & Benefit & Toxicity \\
\hline Leonardi et al. (66) & NSCLC & $N=46$ & $\begin{array}{l}\text { Not reported } \\
13 \% \text { active disease } \\
19 \% \text { receive immune treat }\end{array}$ & Anti PD-1 (no details) & No data & $\begin{array}{l}17 \% \text { flare of baseline disease } \\
26 \% \text { developed other IrAE } \\
\text { No grade } 4-5\end{array}$ \\
\hline Leonardi et al. (67) & NSCLC & $N=56$ & $\begin{array}{l}\text { Rheumatologic } n=25(45 \%) \\
\text { Dermatologic } n=16(29 \%) \\
\text { Endocrine } n=9(16 \%) \\
\text { Crohn/ulcerative colitis } n=6(11 \%) \\
\text { Myastenia/Multiple sclerosis } n=3(5 \%)\end{array}$ & $\begin{array}{l}\text { Nivo } 80 \% \\
\text { Pem } 18 \% \\
\text { Atezo } 2 \%\end{array}$ & $\begin{array}{l}\text { PR 22\% } \\
\text { SD 31\% } \\
\text { PD } 47 \% \\
\text { No relation of flare or steroids at } \\
\text { beginning of treatment with } \\
\text { response }\end{array}$ & $\begin{array}{l}23 \% \text { flare of baseline disease } \\
-13 \% \text { grade } 3,0 \% \text { Grade } 4 \text { and } 5 \\
-50 \% \text { of symptomatic had a flare vs. } 18 \% \text { of } \\
\text { asymptomatic patients } \\
\text { - Rheumatologic patients } 40 \% \text { flare vs. } 10 \% \\
\text { other } \\
38 \% \text { developed another IrAE } \\
5 \% \text { developed both }\end{array}$ \\
\hline Tison et al. (68) & $\begin{array}{l}\text { Melanoma59\% } \\
\text { NSCLC 35\% } \\
\text { Urothelial 3\% } \\
\text { MCC 1.5\% }\end{array}$ & $N=112$ & $\begin{array}{l}\text { Psoriasis } n=31(28 \%) \\
\text { Rheumatoid arthritis } n=20(18 \%) \\
\text { Inflammatory bowel dis } n=14(13 \%) \\
\text { Spondilartritis } n=5(4,5 \%) \\
\text { Lupus } n=7(6,3 \%) \\
\text { Polymyalgia/Gian cell arteritis } n=7 \\
\text { Other } n=28(25 \%)\end{array}$ & $\begin{array}{l}\text { Ipi } n=13 \% \\
\text { Nivo } 56 \% \\
\text { Pem 24\% } \\
\text { Ipi + Nivo 3\% } \\
\text { Atezo 2\% } \\
\text { Ave 2\% }\end{array}$ & $\begin{array}{l}30 \% \mathrm{PR} \\
15 \% \mathrm{CR}\end{array}$ & $\begin{array}{l}47 \% \text { flare of baseline disease } \\
-70 \% \text { mild, } 30 \% \text { severe } \\
-45 \% \text { required steroid } \\
-9 \% \text { other immunosuppression } \\
42 \% \text { another IrAE } \\
-60 \% \text { mild } 40 \% \text { severe } \\
18 \% \text { had both flare + new IrAE } \\
1 \text { death }\end{array}$ \\
\hline \multicolumn{7}{|c|}{ Series including a control group with ICI treated patients } \\
\hline Danlos et al. (69) & $\begin{array}{l}\text { Melanoma } 80 \% \\
\text { NSCLC } 13.3 \% \\
\text { Other } 6.7 \%\end{array}$ & $\begin{array}{l}N=45 \text { vs. } 352 \\
\text { controls }\end{array}$ & $\begin{array}{l}\text { Rheumatic disease, } n=7(13 \%) \\
\text { Dermatologic disease, } n=33(62 \%) \\
\text { Endocrine disease, } n=9(17 \%) \\
\text { Neurologic disease, } n=3(5.7 \%) \\
\text { Hematologic disease, } n=1(1.9 \%) \\
\text { Symptomatic stable disease } 50.9 \% \\
\text { Flare (at ICl initiation) }=5.7 \% \\
6 \text { ICI pretreated experienced colitis }(n=3) \text {, } \\
\text { thyroiditis, hypophisitis, and nephritis }\end{array}$ & $\begin{array}{l}\text { Pem } 75.6 \% \\
\text { Nivo } 22.2 \% \\
\text { Ave } 2.2 \% \\
9 \text { patients pretreated } \\
\text { with AntiCTLA-4/PD-1 }\end{array}$ & Not reported & $\begin{array}{l}20 \text { ( } 44.4 \% \text { ) experienced and IrAE } \\
\text { Of which } 11 \text { (55\%) } \\
\text { where flare UP (i.e., } n=11 / 45-24.5 \%) 10 \\
\text { patients developed a new IrAE } \\
\text { Only in } 5 \text { of the } 20 \text { patients ICI treatment was } \\
\text { stopped } \\
\text { After excluding vitiligo } 51,6 \text { patients did not } \\
\text { develop an IrAE under ICItreatment }\end{array}$ \\
\hline Cortellini et al. (70) & $\begin{array}{l}\text { NSCLC 65.5\% } \\
\text { Melanoma 21\% } \\
\text { RCC 12.5\% } \\
\text { Other1\% }\end{array}$ & $\begin{array}{l}N=85 \text { vs. } 666 \\
\text { controls }\end{array}$ & $\begin{array}{l}\text { Thyroid disorder } n=51 \\
\text { (60\%) Dermatologic } n=14(16.4 \%) \\
\text { Rheumatologic } n=10(11.8 \%) \\
\text { Gastrointestinal/hepatic } n=4(4.7 \%) \\
\text { Neuro-/Nephro- } n=1(1.2 \%) \text { each } \\
\text { Multiple } n=4(4.7 \%)\end{array}$ & No data & $\begin{array}{l}\text { Response rate } 35,3 \% \\
\text { No difference with or without } A D\end{array}$ & $\begin{array}{l}\text { With preexisting AD: } \\
\text {-All grade } 65.9 \%(95 \% \mathrm{Cl} 49.7-85.5) \\
\text {-G3/G4 9.4\% (95\%Cl } 4.1-18.5 \%) \\
\text {-Flare of baseline Disease } 47.1 \% \\
\text {-No Diff between active and inactive disease at } \\
\text { baseline } \\
\text { Without preexisting AD } \\
\text {-All grades 39,9\% (95\%Cl 35.2-45) } \\
\text {-G3/G4 8.8\% (95\%Cl6.7-11.4) }\end{array}$ \\
\hline
\end{tabular}


TABLE 2 | Continued

\begin{tabular}{|c|c|c|c|c|c|c|}
\hline References & Tumor & $N=$ & Baseline autoimmune dis & Drug & Benefit & Toxicity \\
\hline Ricther et al. (71) & $\begin{array}{l}\text { Melanoma 63\% } \\
\text { NSCLC 25\% } \\
\text { Hematologic13\% }\end{array}$ & $\begin{array}{l}N=16 \text { (out of } \\
33 \text { with } \\
\text { preexisting } \mathrm{AD} \\
\text { and } 700 \mathrm{ICl} \\
\text { treated patients) }\end{array}$ & $\begin{array}{l}\text { Polymyalgia rheumatica } n=5(31 \%) \\
\text { Rheumatoid arthritis } n=5(31 \%) \\
\text { Giant Cell arteritis } n=2(13 \%) \\
\text { Sjogren syndrome } n=2(13 \%) \\
\text { Lupus } n=2(13 \%) \\
\text { Ankylosing spondylitis } n=1 \\
\text { Idiopathic aortitis } n=1 \\
\text { Sarcoidosis } n=1 \\
\text { Urticarial vasculitis } n=1\end{array}$ & $\begin{array}{l}\text { Ipi } 31 \% \\
\text { Nivo } 44 \% \\
\text { Pem } 31 \%\end{array}$ & & $\begin{array}{l}\text { IrAE developed in } n=6(38 \%) \\
\text { Colitis } n=3 \text { Only } 1 \text { case had a flare of baseline } \\
\text { Rheumatic Disease } \\
5 \text { of } 6 \text { occurred during first } 4 \text { cycles } \\
4 \text { of } 6 \text { were grade } 3 \text { or } 4 \\
\text { All managed with steroids. One treated with } \\
\text { another IC with no relapse }\end{array}$ \\
\hline Khozin et al. (73) & NSCLC & $\begin{array}{l}538 \text { with AD out } \\
\text { of } 2,425 \text { patients }\end{array}$ & Not reported & Not reported & $\begin{array}{l}\text { No difference for PFS, OS, Time } \\
\text { to treatment discontinuation and } \\
\text { time to next treatment }\end{array}$ & $\begin{array}{l}\text { No increasing IrAE in patients with preexisting } \\
\text { autoimmune disease }\end{array}$ \\
\hline Shah et al. (72) & $\begin{array}{l}\text { Melanoma } 45 \% \\
\text { Lung Cancer } \\
18 \% \\
\text { Other } 37 \%\end{array}$ & $\begin{array}{l}n=22 \text { with } A D \\
\text { out of } 638\end{array}$ & $\begin{array}{l}\text { Hypothyroidism (36.4\%) } \\
\text { Graves disease (9\%) } \\
\text { Psoriasis, vitiligo }(n=2-9 \%) \\
\text { each Ulcerative colitis ( } n=2-9 \%) \\
\text { Type } 1 \text { diabetes, ankylosing spondylitis, lupus, } \\
\text { nephropathy ( } n=1-4.5 \% \text { each) }\end{array}$ & $\begin{array}{l}\text { Ipi } 45 \% \\
\text { Pem } 41 \% \\
\text { Nivo } 27 \%\end{array}$ & No data & $\begin{array}{l}\text { Only } n=4(18 \%) \text { had an exacerbation of } \\
\text { previous Autoimmune disease } \\
19 \text { out of } 22 \text { had a new IrAE } \\
\text { Pulmonary } n=10 \\
\text { Gastrointestinal } n=6 \\
\text { Heptic } n=5 \\
\text { Renal } n=3 \\
\text { Hypothyroidism } n=2 \\
\text { Dermatological } n=2 \\
\text { Musculoskeletal } n=2 \\
\text { Hypophisitis } n=1\end{array}$ \\
\hline Kehl et al. (75) & $\begin{array}{l}\text { Lung cancer } \\
42 \% \\
\text { Melanoma 34\% } \\
\text { Renal 9\% } \\
\text { Urothelial 3.5\% } \\
\text { Head and neck }\end{array}$ & $\begin{array}{l}179 \text { (strict } \\
\text { criteria) } \\
\text { Out of } 4,438 \\
\text { treated patients }\end{array}$ & Not reported & $\begin{array}{l}\text { Nivo } 52 \% \\
\text { Pem } 20 \% \\
\text { Ipi } 21 \% \\
\text { Ipi + Nivo } 5 \% \\
\text { Atezo } 3 \%\end{array}$ & $\begin{array}{l}\text { Mean treatment duration } 13.7 \\
\text { weeks }\end{array}$ & $\begin{array}{l}\text { Pre-existing autoimmune disease not related } \\
\text { with all cause hospitalization but related with } \\
\text { hospitalization for an IrAE (HR } 1.8195 \% \mathrm{Cl} \\
1.21-2.71 \text { ) and prescription of steroids (HR } \\
1.9395 \% \mathrm{Cl} 1.35-2.76 \text { ) }\end{array}$ \\
\hline
\end{tabular}


(Table 2). Concerning antitumor efficacy, there was no difference between patients with or without previous autoimmune disease $(70,73)$. Up to $47 \%$ of patients experienced a flare up of their baseline disease and up to $42 \%$ developed a new IrAE. These figures, of newly generated IrAE, are comparable with those reported from randomized clinical trials (74). Severe and very severe cases (grade 3 and up) were not reported more frequently.

Finally, an analysis of the SEER registry data (75) showed that a preexisting autoimmune disorder is not related to all cause hospitalization but is associated with hospitalization related to the development of IrAE and with steroid prescription.

\section{CONCLUSIONS}

While it is difficult to aggregate such very disparate data some conclusion may still be extracted. First, patients with autoimmune disorders seems to derive the same benefit in term of tumor response compared with people without autoimmune disorders. Conflicting data exist concerning the association between autoimmunity or IrAE and tumor response, with both positive and negative investigations (76-79). Much more research is needed in this area. Thus, there is no an "a priori" reason to deny a potential effective treatment to these patients. Weather this group of patients experience more long-term benefit is still a matter of investigation.

Concerning safety, these patients seem to generate the same spectrum, number, and severity of IrAE (other than the flare up of their baseline autoimmune condition) as patients without preexisting autoimmune disease and thus need to be followedup similarly. The question arises for the flare-up of their autoimmune condition. At least $30 \%$ to a maximum of $80 \%$ of patients, depending on series and type of baseline autoimmune disease, do not experience a flare up. In general, people with skin autoimmune disorders can be managed safely even in case

\section{REFERENCES}

1. Durieux V, Coureau M, Meert AP, Berghmans T, Sculier JP. Autoimmune paraneoplastic syndromes associated to lung cancer: a systematic review of the literature. Lung Cancer. (2017) 106:102-9. doi: 10.1016/j.lungcan.2017.01.015

2. Tivol EA, Borriello F, Schweitzer AN, Lynch WP, Bluestone JA, Sharpe AH. Loss of CTLA-4 leads to massive lymphoproliferation and fatal multiorgan tissue destruction, revealing a critical negative regulatory role of CTLA-4. Immunity. (1995) 3:541-7. doi: 10.1016/1074-7613(95)90125-6

3. Bardhan K, Anagnostou T, Boussiotis VA. The PD1:PD-L1/2 pathway from discovery to clinical implementation. Front Immunol. (2016) 7:550. doi: 10.3389/fimmu.2016.00550

4. Schubert D, Bode C, Kenefeck R, Hou TZ, Wing JB, Kennedy A, et al. Autosomal dominant immune dysregulation syndrome in humans with CTLA4 mutations. Nat Med. (2014) 20:1410-6. doi: 10.1038/nm.3746

5. Kuehn HS, Ouyang W, Lo B, Deenick EK, Niemela JE, Avery DT, et al. Immune dysregulation in human subjects with heterozygous germline mutations in CTLA4. Science. (2014) 345:1623-7. doi: 10.1126/science. 1255904

6. Fife BT, Pauken KE. The role of the PD-1 pathway in autoimmunity and peripheral tolerance. Ann N Y Acad Sci. (2011) 1217:4559. doi: 10.1111/j.1749-6632.2010.05919.x

7. Khan SA, Pruitt SL, Xuan L, Gerber DE. Prevalence of autoimmune disease among patients with lung cancer: implications for immunotherapy of very severe flares (50). The same apply for those with thyroid dysfunction but severe hyperthyroidism can be encountered even in previously chronic hypothyroid patients (55).

Some data exist for patients with rheumatic disease, and it seems that at least $50 \%$ of them experience a disease flare. Grade 4 and 5 flare-ups seems infrequent and generally are manageable with steroids and anti-TNF medications. These patients can receive checkpoint inhibitors provided that no best option is available, they are informed on the potential disease flare and a careful follow-up is instituted.

Insufficient data exist for patients with inflammatory bowel disease. When these manifestations develop during a previous anti CTLA-4 treatment, the relapse rate under anti PD-1/PDL1 therapy seems low and it is probable that these patients can be safely treated.

There is a need for more research in groups of patients with inflammatory bowel disease which receive checkpoint inhibitors for the first time. Their administration clearly poses some risks, but it needs to be evaluated taking into account the effectiveness of available alternatives and the patient should understand the potential benefits and harms as well as the risk -benefit ratio.

Based in SEER data, administration of immune checkpoint inhibitors do not result in excess hospitalizations compared to similar patients without autoimmune disease but the hospitalizations are strongly related to the development or flare up of autoimmune conditions. An observational trial concerning ipilimumab in patients with melanoma and underlying autoimmune diseases (80) has been performed but, to our best knowledge, results have not yet been reported.

\section{AUTHOR CONTRIBUTIONS}

All authors contributed to literature search, data extraction, and drafting of manuscript.

treatment options. JAMA Oncol. (2016) 2:1507-8. doi: 10.1001/jamaoncol.201 6.2238

8. Sculier C, Bentea G, Ruelle L, Grigoriu B, Coureau M, Gorham $\mathrm{J}$, et al. Autoimmune paraneoplastic syndromes associated to lung cancer: a systematic review of the literature: part 5: neurological autoantibodies, discussion, flow chart, conclusions. Lung Cancer. (2017) 111:16475. doi: 10.1016/j.lungcan.2017.07.026

9. Ruelle L, Bentea G, Sideris S, El Koulali M, Holbrechts S, Lafitte JJ, et al. Autoimmune paraneoplastic syndromes associated to lung cancer: a systematic review of the literature part 4: neurological paraneoplastic syndromes, involving the peripheral nervous system and the neuromuscular junction and muscles. Lung Cancer. (2017) 111:150-63. doi: 10.1016/j.lungcan.2017. 07.025

10. Holbrechts S, Gorham J, Sideris S, Meert AP, Durieux V, Berghmans $\mathrm{T}$, et al. Autoimmune paraneoplastic syndromes associated to lung cancer: A systematic review of the literature: part 2: hematologic, cutaneous and vascular syndromes. Lung Cancer. (2017) 106:93-101. doi: 10.1016/j.lungcan.2017.01.016

11. Bentea G, Sculier C, Grigoriu B, Meert AP, Durieux V, Berghmans T, et al. Autoimmune paraneoplastic syndromes associated to lung cancer: a systematic review of the literature: part 3: neurological paraneoplastic syndromes, involving the central nervous system. Lung Cancer. (2017) 106:83-92. doi: 10.1016/j.lungcan.2017.01.017 
12. Hasan Ali O, Berner F, Bomze D, Fässler M, Diem S, Cozzio A, et al. Human leukocyte antigen variation is associated with adverse events of checkpoint inhibitors. Eur J Cancer. (2019) 107:8-14. doi: 10.1016/j.ejca.2018.11.009

13. Park HK, Ahn M, Park M, Lee S. The HLA-DRB1 polymorphism is associated with atopic dermatitis, but not egg allergy in korean children. Allergy Asthma Immunol Res. (2012) 4:143-9. doi: 10.4168/aair.2012.4.3.143

14. Kongkaew S, Rungrotmongkol T, Punwong C, Noguchi H, Takeuchi $\mathrm{F}$, Kungwan $\mathrm{N}$, et al. Interactions of HLA-DR and topoisomerase I epitope modulated genetic risk for systemic sclerosis. Sci Rep. (2019) 9:745. doi: 10.1038/s41598-018-37038-z

15. Ombrello MJ, Remmers EF, Tachmazidou I, Grom A, Foell DJ, Haas P, et al. HLA-DRB $1^{*} 11$ and variants of the MHC class II locus are strong risk factors for systemic juvenile idiopathic arthritis. Proc Natl Acad Sci USA. (2015) 112:15970-5. doi: 10.1073/pnas.1520779112

16. Goyette P, Boucher G, Mallon D, Ellinghaus E, Jostins L, Huang H, et al. Highdensity mapping of the MHC identifies a shared role for HLA-DRB1*01:03 in inflammatory bowel diseases and heterozygous advantage in ulcerative colitis. Nat Genet. (2015) 47:172-9. doi: 10.1038/ng.3176

17. Chat V, Ferguson R, Simpson D, Kazlow E, Lax R, Moran U, et al. Autoimmune genetic risk variants as germline biomarkers of response to melanoma immune-checkpoint inhibition. Cancer Immunol Immunother. (2019) 68:897-905. doi: 10.1007/s00262-019-02318-8

18. Hoefsmit EP, Rozeman EA, Haanen J, Blank CU. Susceptible loci associated with autoimmune disease as potential biomarkers for checkpoint inhibitor-induced immune-related adverse events. ESMO Open. (2019) 4:e000472. doi: 10.1136/esmoopen-2018-000472

19. Yoneshima Y, Tanaka K, Shiraishi Y, Hata K, Watanabe H, Harada T, et al. Safety and efficacy of PD-1 inhibitors in non-small cell lung cancer patients positive for antinuclear antibodies. Lung Cancer. (2019) 130:59. doi: 10.1016/j.lungcan.2019.01.014

20. Sakakida T, Ishikawa T, Chihara Y, Harita S, Uchino J, Tabuchi Y, et al. Safety and efficacy of PD-1/PD-L1 blockade in patients with preexisting antinuclear antibodies. Clin Transl Oncol. (2019). doi: 10.1007/s12094-019-02214-8

21. Mackintosh D, Islam MF, Ng J, Basham J. Immune checkpoint inhibitor use in antisynthetase syndrome. Asia Pacific J Clin Oncol. (2019) 15:2669. doi: 10.1111/ajco.13141

22. Audemard A, de Raucourt S, Miocque S, Comoz F, Giraud JM, Dreno B, et al. Melanoma-associated retinopathy treated with ipilimumab therapy. Dermatology. (2013) 227:146-9. doi: 10.1159/000353408

23. Aya F, Ruiz-Esquide V, Viladot M, Font C, Prieto-Gonzalez S, Prat A, et al. Vasculitic neuropathy induced by pembrolizumab. Ann Oncol. (2017) 28:4334. doi: 10.1093/annonc/mdw613

24. Beck KM, Dong J, Geskin LJ, Beltrani VP, Phelps RG, Carvajal RD, et al. Disease stabilization with pembrolizumab for metastatic acral melanoma in the setting of autoimmune bullous pemphigoid. J Immunother Cancer. (2016) 4:20. doi: 10.1186/s40425-016-0123-3

25. Benson Z, Gordon S, Nicolato P, Poklepovic A. Immunotherapy for metastatic melanoma with right atrial involvement in a patient with rheumatoid arthritis. Case Rep Oncol Med. (2017) 2017:8095601. doi: 10.1155/2017/80 95601

26. Bostwick AD, Salama AK, Hanks BA. Rapid complete response of metastatic melanoma in a patient undergoing ipilimumab immunotherapy in the setting of active ulcerative colitis. J Immunother Cancer. (2015) 3:19. doi: 10.1186/s40425-015-0064-2

27. Chia PL, John T. Severe psoriasis flare after anti-programmed death ligand 1 (PD-L1) therapy for metastatic non-small cell lung cancer (NSCLC). J Immunother. (2016) 39:202-4. doi: 10.1097/CJI.0000000000000121

28. Cooper DS, Meriggioli MN, Bonomi PD, Malik R. Severe exacerbation of myasthenia gravis associated with checkpoint inhibitor immunotherapy. $J$ Neuromuscul Dis. (2017) 4:169-73. doi: 10.3233/JND-170219

29. Esfahani K, Miller WH Jr. Reversal of autoimmune toxicity and loss of tumor response by interleukin-17 blockade. N Engl J Med. (2017) 376:198991. doi: 10.1056/NEJMc1703047

30. Frohne CC, Llano EM, Perkovic A, Cohen RD, Luke JJ. Complete response of metastatic melanoma in a patient with Crohn's disease simultaneously receiving anti-alpha4beta7 and anti-PD1 antibodies. J Immunother Cancer. (2019) 7:1. doi: 10.1186/s40425-018-0484-x
31. Gerdes LA, Held K, Beltran E, Berking C, Prinz JC, Junker A, et al. CTLA4 as immunological checkpoint in the development of multiple sclerosis. Ann Neurol. (2016) 80:294-300. doi: 10.1002/ana.24715

32. Gettings EJ, Hackett CT, Scott TF. Severe relapse in a multiple sclerosis patient associated with ipilimumab treatment of melanoma. Mult Scler. (2015) 21:670. doi: 10.1177/1352458514549403

33. Gielisse EA, de Boer NK. Ipilimumab in a patient with known Crohn's disease: to give or not to give? J Crohns Colitis. (2014) 8:1742. doi: 10.1016/j.crohns.2014.08.002

34. Hegde U, Chauhan A, Desai N. Myasthenic syndrome with pembrolizumab use. Neurology. (2017) 88(Suppl P6.175):1526-632X.

35. Kageyama SI, Yamaguchi S, Ito S, Suehara Y, Saito T, Akaike K, et al. A case report of using nivolumab for a malignant melanoma patient with rheumatoid arthritis. Int Cancer Conf J. (2016) 5:192-6. doi: 10.1007/s13691-016-0256-8

36. Kamil F, Student M, Cohen M, Kumta N, Wan D. Ipilimumab-induced colitis in a patient with ulcerative colitis and lung cancer: 1361. Am J Gastroenterol. (2013) 108:S404. doi: 10.14309/00000434-201310001-01361

37. Kato Y, Otsuka A, Miyachi Y, Kabashima K. Exacerbation of psoriasis vulgaris during nivolumab for oral mucosal melanoma. J Eur Acad Dermatol Venereol. (2016) 30:e89-91. doi: 10.1111/jdv.13336

38. Kyi C, Carvajal RD, Wolchok JD, Postow MA. Ipilimumab in patients with melanoma and autoimmune disease. J Immunother Cancer. (2014) 2:35. doi: 10.1186/s40425-014-0035-Z

39. Lau KH, Kumar A, Yang IH, Nowak RJ. Exacerbation of myasthenia gravis in a patient with melanoma treated with pembrolizumab. Muscle Nerve. (2016) 54:157-61. doi: 10.1002/mus.25141

40. Maeda O, Yokota K, Atsuta N, Katsuno M, Akiyama M, Ando Y. Nivolumab for the treatment of malignant melanoma in a patient with pre-existing myasthenia gravis. Nagoya J Med Sci. (2016) 78:119-22.

41. Matsumura N, Ohtsuka M, Kikuchi N, Yamamoto T. Exacerbation of psoriasis during nivolumab therapy for metastatic melanoma. Acta Derm Venereol. (2016) 96:259-60. doi: 10.2340/00015555-2212

42. Maul LV, Weichenthal M, Kahler KC, Hauschild A. Successful anti-PD-1 antibody treatment in a metastatic melanoma patient with known severe autoimmune disease. I Immunother. (2016) 39:188-90. doi: 10.1097/CJI.0000000000000118

43. Nabel CS, Severgnini M, Hung YP, Cunningham-Bussel A, Gjini E, Kleinsteuber K, et al. Anti-PD-1 immunotherapy-induced flare of a known underlying relapsing vasculitis mimicking recurrent cancer. Oncologist. (2019) 24:1013-21. doi: 10.1634/theoncologist.2018-0633

44. Narita T, Oiso N, Taketomo Y, Okahashi K, Yamauchi K, Sato M, et al. Serological aggravation of autoimmune thyroid disease in two cases receiving nivolumab. J Dermatol. (2016) 43:210-4. doi: 10.1111/1346-8138.13028

45. Pedersen M, Andersen R, Norgaard P, Jacobsen S, Thielsen P, Thor Straten $\mathrm{P}$, et al. Successful treatment with Ipilimumab and Interleukin-2 in two patients with metastatic melanoma and systemic autoimmune disease. Cancer Immunol Immunother. (2014) 63:1341-6. doi: 10.1007/s00262-014-1607-y

46. Phadke SD, Ghabour R, Swick BL, Swenson A, Milhem M, Zakharia Y. Pembrolizumab therapy triggering an exacerbation of preexisting autoimmune disease: a report of 2 patient cases. J Investig Med High Impact Case Rep. (2016) 4:2324709616674316. doi: 10.1177/2324709616674316

47. Plachouri K, Mohr M, Sunderkoetter C, Weishaupt C. Induction of muscular sarcoidosis in a metastastic melanoma patient treated with ipilimumab. Journal der Deutschen Dermatologischen Gesellschaft. (2012) 10:861.

48. Puri A, Homsi J. The safety of pembrolizumab in metastatic melanoma and rheumatoid arthritis. Melanoma Res. (2017) 27:519-23. doi: 10.1097/CMR.0000000000000387

49. Roche L, Murphy M, Power D. Treatment of Merkel cell carcinoma with pembrolizumab in a patient with psoriasis and psoriatic arthritis. $\mathrm{Br} J$ Dermatol. (2017) 177:143. doi: 10.1111/jdv.02-14275

50. Sahuquillo-Torralba A, Ballester-Sanchez R, Pujol-Marco C, Botella-Estrada R. Pembrolizumab: a new drug that can induce exacerbations of psoriasis. Actas Dermosifiliogr. (2016) 107:264-6. doi: 10.1016/j.ad.2015.07.012

51. Stephen Bagley J, Kosteva JA, Evans TL, Langer CJ. Immune thrombocytopenia exacerbated by nivolumab in a patientwith non-small-cell lung cancer. Cancer Treat Commun. (2016) 6:20-3. doi: 10.1016/j.ctrc.2016.02.009 
52. Tagliamento M, Grossi F, Paolino S, Rijavec E, Genova C, Rossi G, et al. Nivolumab treatment in advanced lung cancer patient with chronic active hepatitis C and systemic lupus erythematosus. Immunotherapy. (2019) 11:873-9. doi: 10.2217/imt-2019-0025

53. Uemura M, Trinh VA, Haymaker C, Jackson N, Kim DW, Allison JP, et al. Selective inhibition of autoimmune exacerbation while preserving the anti-tumor clinical benefit using IL- 6 blockade in a patient with advanced melanoma and Crohn's disease: a case report. J Hematol Oncol. (2016) 9:81. doi: 10.1186/s13045-016-0309-7

54. Weishaupt CMM, Sunderkötter C. Muscular sarcoidosis in a metastastic melanoma patient treated with ipilimumab. Journal der Deutschen Dermatologischen Gesellschaft. (2012) 10:683.

55. Yonezaki K, Kobayashi T, Imachi H, Yoshimoto T, Kikuchi F, Fukunaga K, et al. Combination therapy of ipilimumab and nivolumab induced thyroid storm in a patient with Hashimoto's disease and diabetes mellitus: a case report. J Med Case Rep. (2018) 12:171. doi: 10.1186/s13256-018-1708-x

56. Zaremba A, Chorti E, Jockenhofer F, Bolz S, Sirin S, Glas M, et al. Metastatic Merkel cell carcinoma and myasthenia gravis: contraindication for therapy with immune checkpoint inhibitors? J Immunother Cancer. (2019) 7:141. doi: 10.1186/s40425-019-0626-9

57. Zhu J, Li Y. Myasthenia gravis exacerbation associated with pembrolizumab. Muscle Nerve. (2016) 54:506-7. doi: 10.1002/mus.25055

58. De Bock M, Hulstaert E, Kruse V, Brochez L. Psoriasis vulgaris exacerbation during treatment with a PD-1 checkpoint inhibitor: case report and literature review. Case Rep Dermatol. (2018) 10:190-7. doi: 10.1159/000491572

59. Akturk HK, Alkanani A, Zhao Z, Yu L, Michels AW. PD-1 inhibitor immunerelated adverse events in patients with preexisting endocrine autoimmunity. $J$ Clin Endocrinol Metab. (2018) 103:3589-92. doi: 10.1210/jc.2018-01430

60. Weinstock C, Singh H, Maher VE, Kim G, Pazdur R. FDA analysis of patients with baseline autoimmune diseases treated with PD-1/PD-L1 immunotherapy agents. J Clin Oncol. (2017) 35:3018. doi: 10.1200/JCO.2017.35.15_suppl.3018

61. Lee B, Wong A, Kee D, Neeson P, Shackleton M, McArthur G, et al. The use of ipilimumab in patients with rheumatoid arthritis and metastatic melanoma. Ann Oncol. (2016) 27:1174-7. doi: 10.1093/annonc/mdw056

62. Menzies AM, Johnson DB, Ramanujam S, Atkinson VA, Wong NM, et al. Anti-PD-1 therapy in patients with advanced melanoma and preexisting autoimmune disorders (AD) or major toxicity with ipilimumab (Ipi). J Clin Oncol. (2016) 34:9515. doi: 10.1200/JCO.2016.34.15_suppl.9515

63. Johnson DB, Sullivan RJ, Ott PA, Carlino MS, Khushalani NI, Ye $\mathrm{F}$, et al. Ipilimumab therapy in patients with advanced melanoma and preexisting autoimmune disorders. JAMA Oncol. (2016) 2:23440. doi: 10.1001/jamaoncol.2015.4368

64. Kähler KC, Eigentler TK, Gesierich A, Heinzerling L, Loquai C, Meier $\mathrm{F}$, et al. Ipilimumab in metastatic melanoma patients with pre-existing autoimmune disorders. Cancer Immunol Immunother. (2018) 67:82534. doi: 10.1007/s00262-018-2134-Z

65. Gutzmer R, Koop A, Meier F, Hassel JC, Terheyden P, Zimmer L, et al. Programmed cell death protein-1 (PD-1) inhibitor therapy in patients with advanced melanoma and preexisting autoimmunity or ipilimumab-triggered autoimmunity. Eur J Cancer. (2017) 75:24-32. doi: 10.1016/j.ejca.2016.12.038

66. Leonardi GC, Gainor JF, Azimi RS, Riess J, Rizvi H, Hellmann MD, et al. Use of PD-1 pathway inhibitors among patients with non-small cell lung cancer (NSCLC) and preexisting autoimmune disorders. J Clin Oncol. (2017) 35:9081. doi: 10.1200/JCO.2017.35.15_suppl. 9081

67. Leonardi GC, Gainor JF, Altan M, Kravets S, Dahlberg SE, Gedmintas L, et al. Safety of programmed death-1 pathway inhibitors among patients with nonsmall-cell lung cancer and preexisting autoimmune disorders. J Clin Oncol. (2018) 36:1905-12. doi: 10.1200/JCO.2017.77.0305

68. Tison A, Quere G, Misery L, Funck-Brentano E, Danlos FX, Routier E, et al. Safety and efficacy of immune checkpoint inhibitors in patients with cancer and preexisting autoimmune disease: a nationwide, multicenter cohort study. Arthrit Rheumatol. (2019) 71:2100-11. doi: 10.1136/annrheumdis-2018-eular. 5840
69. Danlos FX, Voisin AL, Dyevre V, Michot JM, Routier E, Taillade L, et al. Safety and efficacy of anti-programmed death 1 antibodies in patients with cancer and pre-existing autoimmune or inflammatory disease. Eur J Cancer. (2018) 91:21-9. doi: 10.1016/j.ejca.2017.12.008

70. Cortellini A, Buti S, Santini D, Perrone F, Giusti R, Tiseo M, et al. Clinical outcomes of patients with advanced cancer and preexisting autoimmune diseases treated with anti-programmed death-1 immunotherapy: a real-world transverse study. Oncologist. (2019) 24:e327-37. doi: 10.1634/theoncologist.2018-0618

71. Richter MD, Pinkston O, Kottschade LA, Finnes HD, Markovic SN, Thanarajasingam U. Brief report: cancer immunotherapy in patients with preexisting rheumatic disease: the mayo clinic experience. Arthrit Rheumatol. (2018) 70:356-60. doi: 10.1002/art.40397

72. Shah M, Jizzini M, Majzoub I, Qdaisat A, Reyes-Gibby C, Yeung SC. Safety of immune checkpoint blockade in patients with cancer and preexisting autoimmune diseases and/or chronic inflammatory disorders. J Immunother Precis Oncol. (2019) 2:59-64. doi: 10.4103/JIPO.JIPO_11_19

73. Khozin S, Walker MS, Jun M, Chen L, Stepanski E, Rubinstein WS, et al. Real-world outcomes of patients with advanced nonsmall cell lung cancer (aNSCLC) and autoimmune disease (AD) receiving immune checkpoint inhibitors (ICIs). J Clin Oncol. (2019) 37:110. doi: 10.1200/JCO.2019.37.15_suppl.110

74. Haanen J, Carbonnel F, Robert C, Kerr KM, Peters S, Larkin J, et al. Management of toxicities from immunotherapy: ESMO Clinical Practice Guidelines for diagnosis, treatment and follow-up. Ann Oncol. (2017) 28:iv119-42. doi: 10.1093/annonc/mdx225

75. Kehl KL, Yang S, Awad MM, Palmer N, Kohane IS, Schrag D. Preexisting autoimmune disease and the risk of immune-related adverse events among patients receiving checkpoint inhibitors for cancer. Cancer Immunol Immunother. (2019) 68:917-26. doi: 10.1007/s00262-019-0 2321-z

76. Haratani K, Hayashi H, Chiba Y, Kudo K, Yonesaka K, Kato R, et al. Association of immune-related adverse events with nivolumab efficacy in non-small-cell lung cancer. JAMA Oncol. (2018) 4:3748. doi: 10.1001/jamaoncol.2017.2925

77. Freeman-Keller M, Kim Y, Cronin H, Richards A, Gibney G, Weber JS. Nivolumab in resected and unresectable metastatic melanoma: characteristics of immune-related adverse events and association with outcomes. Clin Cancer Res. (2016) 22:886-94. doi: 10.1158/1078-0432.CCR-15-1136

78. Postow MA, Sidlow R, Hellmann MD. Immune-related adverse events associated with immune checkpoint blockade. N Engl J Med. (2018) 378:15868. doi: 10.1056/NEJMra1703481

79. Attia P, Phan GQ, Maker AV, Robinson MR, Quezado MM, Yang JC, et al. Autoimmunity correlates with tumor regression in patients with metastatic melanoma treated with anti-cytotoxic T-lymphocyte antigen-4. J Clin Oncol. (2005) 23:6043-53. doi: 10.1200/JCO.2005. 06.205

80. Squibb BM. Study of Autoimmune Disease Complications Following Ipilimumab Treatment Among Melanoma Patients With Underlying Autoimmune Diseases. ClinicalTrials.gov Identifier: NCT02739386 (2019). Available online at: https://clinicaltrials.gov/ct2/show/NCT02739386 (accessed April 14, 2020).

Conflict of Interest: The authors declare that the research was conducted in the absence of any commercial or financial relationships that could be construed as a potential conflict of interest.

Copyright () 2020 Coureau, Meert, Berghmans and Grigoriu. This is an open-access article distributed under the terms of the Creative Commons Attribution License (CC BY). The use, distribution or reproduction in other forums is permitted, provided the original author(s) and the copyright owner(s) are credited and that the original publication in this journal is cited, in accordance with accepted academic practice. No use, distribution or reproduction is permitted which does not comply with these terms. 